\title{
Listening to parents: The role of symptom perception in pediatric palliative home care
}

\author{
RENÉ VOLLENBROICH, M.D., M.P.P., ${ }^{1,2}$ GIAN DOMENICO BORASIO, M.D., ${ }^{3}$ \\ AYDA DUROUX, M.D., ${ }^{1}$ MONIKA GRASSER, M.D., ${ }^{1}$ MONIKA BRANDSTÄTTER, M.A., ${ }^{4}$ \\ AND MONIKA FÜHRER, M.D. ${ }^{1}$ \\ ${ }^{1}$ Coordination Center for Pediatric Palliative Care, Dr. von Haunersches Kinderspital, Ludwig-Maximilians-University, \\ Munich, Germany \\ ${ }^{2}$ Department of Cardiology, University Hospital Bern, Berne, Switzerland \\ ${ }^{3}$ Service de Soins Palliatifs, Centre Hospitalier Universitaire Vaudois (CHUV), University of Lausanne, \\ Lausanne, Switzerland \\ ${ }^{4}$ Interdisciplinary Center for Palliative Medicine, Ludwig-Maximilians-University Munich, Munich, Germany \\ (Received February 1, 2015; AcCePted April 22, 2015)
}

\begin{abstract}
Objective: This study analyzes symptom perception by parents and healthcare professionals and the quality of symptom management in a pediatric palliative home care setting and identifies which factors contribute to a high quality of palliative and end-of-life care for children.

Methods: In this retrospective, cross-sectional study, parents were surveyed at the earliest three months after their child's death. All children were cared for by a specialized home pediatric palliative care team that provides a 24/7 medical on-call service. Questionnaires assessed symptom prevalence and intensity during the child's last month of life as perceived by parents, symptom perception, and treatment by medical staff. The responses were correlated with essential palliative care outcome measures (e.g., satisfaction with the care provided, qualityof-life of affected children and parents, and peacefulness of the dying phase).

Results: Thirty-eight parent dyads participated (return rate $84 \%$; 35\% oncological disorders). According to parental report, dyspnea (61\%) and pain (58\%) were the dominant symptoms with an overall high symptom load (83\%). Pain, agitation, and seizures could be treated more successfully than other symptoms. Successful symptom perception was achieved in most cases and predicted the quality of symptom treatment $\left(R^{2}, 0.612\right)$. Concordant assessment of symptom severity between parents and healthcare professionals (HCPs) improved the satisfaction with the care provided ( $p=0.037)$ as well as the parental quality-of-life $(p=0.041)$. Even in cases with unsuccessful symptom control, parents were very satisfied with the SHPPC team's care (median 10; numeric rating scale 0-10) and rated the child's death as highly peaceful (median 9).

Significance of the results: The quality and the concordance of symptom perception between parents and HCPs essentially influence parental quality-of-life as well as parental satisfaction and constitute a predictive factor for the quality of symptom treatment and palliative care.
\end{abstract}

KEYWORDS: Pediatric palliative home care, Symptom perception, End-of-life symptoms

\section{INTRODUCTION}

Since 2009, a significant number of specialized home pediatric palliative care (SHPPC) teams have been implemented in Germany in order to make high

Address correspondence and reprint requests to: René Vollenbroich, Department of Cardiology, Inselspital Bern, University of Berne, Murtenstrasse 5, 3008 Berne, Switzerland. E-mail: rene_ vollenbroich@post.harvard.edu quality palliative home care for children and adolescents possible (Bona et al., 2011; Friedrichsdorf et al., 2007; Pierucci et al., 2001; Toce et al., 2003). As defined by the World Health Organization, pediatric palliative care comprehensively addresses the physical, psychosocial, and spiritual needs of the children and their families (WHO, 1998). Recent studies have shown that all these factors need to be considered in an orchestrated effort to devise an effective strategy 
of care (Dangel, 2002; Hilden et al., 2001; Moody et al., 2011; Swinney et al., 2007). Within this strategy, the provision of optimal symptom management plays an important role for affected children, their families, and all care providers involved (Drake et al., 2003; Galloway et al., 2000; Himelstein et al., 2004; Zhukovsky et al., 2009).

Most of the existing data on pediatric palliative care relate to oncological cohorts in hospital settings and in particular to the treatment of pain (Carter et al., 2004; Liben et al., 2008; Morgan et al., 2000). However, recent experience of pediatric palliative care teams shows that a significant proportion of children with palliative care needs suffer from congenital disorders or diseases acquired during the perinatal period. Effective symptom management for all patient groups requires a deeper understanding of all possible symptoms that might occur in addition to a comprehensive analysis of the various factors influencing the well-being of the child and the family, be it in a clinical or home setting (Kreicbergs et al., 2005; Poltorak et al., 2006).

Wolfe et al. (2000) found a significant disparity between parents and healthcare providers when reporting on the occurrence and severity of symptoms. They postulate that unsuccessful symptom treatment might be the result of a lack of recognition and perception (Wolfe et al., 2000). Thus, distinguishing between symptom perception and the effect of symptom treatment by the medical team during the provision of palliative care could be of considerable importance.

The goal of this study was to evaluate the perception of the kind and severity of symptoms by parents and HCPs, and the success of symptom treatment, as well as their influence on the quality of palliative care provided by a SHPPC team as perceived by the parents.

\section{METHODS}

\section{Study Site}

This study was part of a comprehensive retrospective, cross-sectional study (Vollenbroich et al., 2012) performed from June to December 2007 at the Coordination Center for Pediatric Palliative Care (CPPC) of the University of Munich, Germany. The CPPC hosts a multi-professional SHPPC team that was implemented in 2004 to provide palliative home care for children, adolescents, and young adults with life limiting diseases in southeastern Bavaria (population of about 4.5 million). The main tasks of the team include the coordination of professional assistance, the provision of palliative care in cooperation with local healthcare professionals (HCPs), and a 24/7 medical on-call service. The study was approved by the Research Ethics Committee of the Ludwig-Maximilians-University of Munich, Germany.

\section{Participants}

Eligible participants were all families that lost a child at least three months prior to the start of the study due to a life-limiting disease and had received home palliative care through the SHPPC team between March 2004 and March 2007. Their main SHPPC team contact informed them by phone of the purpose and methods of the study. Families who agreed to participate were sent information material, informed consent forms, the questionnaire and a return envelope. Exclusion criteria comprised parents' inadequate language proficiency or mental disorder, missing contact data, only one-time contact with the SHPPC team, and pre- or perinatal deaths.

\section{Parental Questionnaire}

The questionnaire comprises a total of 56 items, and was developed from a comprehensive literature search, review of validated tools, and clinical experience. It assesses socio-demographic data about the respondents (8 items), information about the child's condition (4 items), satisfaction with specific aspects of the care provided by the SHPPC team (22 items), quality of care before and after SHPPC team involvement (12 items), and satisfaction with the dying phase (10 items). The dying phase was defined as the phase in which the members of the multi-professional team caring for the patient agreed that the patient was likely to die (Ellershaw et al., 2003).

In addition, parents were asked for any general comments or suggestions. For most items, numeric rating scales (NRS, 0-10) were used. The parents' current quality-of-life was assessed through a 1-item NRS (0-10), and the parents were also asked to rate their child's quality-of-life before and after SHPPC team involvement using the same scale. The questionnaire was piloted in four families and adapted according to feedback. The parents were advised to contact the SHPPC team for support if they developed psychological distress after completing the questionnaire.

To assess symptom perception and treatment, the parents were asked to list up to four main symptoms, from which their child suffered the most during his/ her last month of life. Subsequently, four-point Likert scales were used to evaluate symptom severity (mild, moderate, strong, very strong) as well as quality of symptom perception and treatment (strongly disagree, somewhat disagree, somewhat agree, strongly agree) by the SHPPC team.

In order to categorize successful and unsuccessful symptom perception and treatment, the answers 
were dichotomized (strongly/somewhat disagree and strongly/somewhat agree). Dichotomized answers were used for further statistical analysis. If more than four symptoms were reported, only the four most severe symptoms were taken into account. The original questionnaire is available from the authors on request.

\section{Healthcare Professionals' Chart Review}

In addition to the parental questionnaire, a chart review of all patients enrolled was performed in order to determine if parents and SHPPC team members perceived the same symptoms and if they consistently classified symptom strength as well as the quality of treatment.

\section{Statistical Analysis}

Descriptive statistics (\%, mean $\pm \mathrm{SD}$, median, and/or range) were calculated for symptom distribution, severity, perception, treatment, and quality-of-care. For graded variables via Likert scales, a two-tailed Fisher's exact test was used to compare dichotomized answers. Pearson correlations and multivariate analyses were used to assess the relationships of presence and severity of symptoms, symptom perception, and symptom treatment, with measures of care (e.g., 24/7 service, general quality-of-care, quality-oflife of children/parents, peacefulness of the dying phase, etc.). In addition, Pearson correlations and multivariate analyses were used to estimate predictive values for the quality of symptom treatment. The significance level was set at $p<0.05$. Bonferroni correction of $p$-values for multiple comparisons was performed. SPSS version 20.0 was used to analyze the data.

\section{RESULTS}

\section{Study Participants}

Out of 106 consecutive patients, 62 parent dyads fulfilled the eligibility criteria (44 children were alive and still cared for by the SHPPC team). Seventeen couples were excluded according to the exclusion criteria (missing contact data $(n=6)$, one-time contact $(n=5)$, inadequate language proficiency $(n=2)$, and pre- or perinatal deaths $(n=4))$. Of the remaining 45 couples, $43(96 \%)$ agreed to participate. The questionnaire was returned by 38 out of 45 parent sets (response rate, $84 \%$ ). In 23 families, both mother and father completed the questionnaire with shared answers. In one case, only the father completed the questionnaire. In the remaining 14 families, the mothers completed the questionnaire.
The majority of parents ( $82 \%)$ were married and 31 to 40 years old (66\%), $20 \%$ of the families were immigrants. The highest education level was elementary school in $7 \%$, secondary school in $22 \%$, high school in $3 \%$, and university education in $29 \%$.

Of the 38 deceased children, 24 were male, median age was 3.4 years $(0-34.3)$. In one case, the patient died at age 34 but was taken care of by the SHPPC team because of his developmental impairment caused by an inborn error of metabolism. Underlying diseases included congenital (36\%, including congenital heart disease), oncological (35\%), neurological (13\%), cardiac (9\%), and other disorders (7\%). $71 \%$ of the children died at home ( $81 \%$ of those $\geq 1$ year of age).

The interval between the child's death and the survey ranged from 3 to 37 months (median $=19.5$ months). The duration of SHPPC team's care ranged from $1-48$ weeks $($ median $=6.5$ weeks $)$ and included $2-$ 79 contacts (median $=30$ ). In six cases, nutrition was supported via a percutaneous endoscopic gastrostomy. Advanced pain management (e.g., multi-disciplinary pain managing approach, provision of patient-controlled analgesia pumps) was provided by the SHPPC team in 22 cases.

\section{Symptom Perception by Parents}

According to the parental reports, dyspnea (61\%) and pain $(58 \%)$ were the main symptoms from which the children were suffering in their last month of life, followed by nausea/vomiting (34\%), and agitation (34\%). Overall, $82 \%$ of symptoms were described as severe. "Poor appetite," "fatigue," and "other nonneurological symptoms" were constantly assessed with the highest grade of severity (see Fig. 1). The

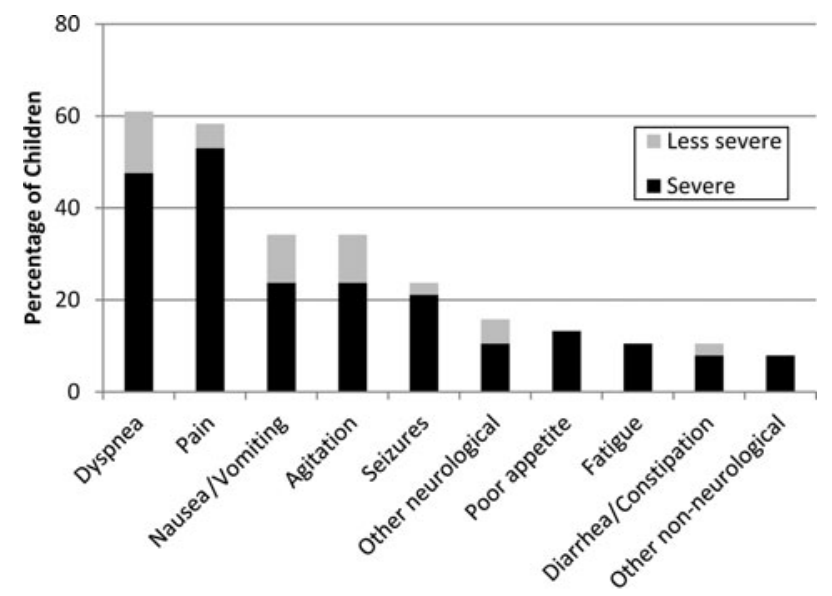

Fig. 1. Distribution and severity of children's symptoms in their last month of life according to parental report $(n=38)$. "Other neurological symptoms" includes spasticity, neurogenic urinary retention, blindness, and hearing loss. "Other non-neurological symptoms" includes pyrexia, fecal incontinence, gastro-oesophageal reflux, and lymphedema. 
Table 1. Concordance between parental and SHPPC team's assessment of symptom severity

\begin{tabular}{lccr}
\hline \hline & \multicolumn{3}{c}{ Symptom Severity } \\
\cline { 2 - 4 } & Severe & Less severe & Abs. \% \\
\hline Dyspnea & $15(18)$ & $8(5)$ & 73 \\
Pain & $20(20)$ & $2(2)$ & 100 \\
Nausea/Vomiting & $7(9)$ & $6(4)$ & 72 \\
Agitation & $9(9)$ & $4(4)$ & 100 \\
Seizures & $7(8)$ & $2(1)$ & 68 \\
Other neurological & $3(4)$ & $3(2)$ & 71 \\
Poor appetite & $3(5)$ & $2(0)$ & 30 \\
Fatigue & $3(4)$ & $1(0)$ & 38 \\
Diarrhea / Constipation & $2(3)$ & $2(1)$ & 58 \\
Other non-neurological & $4(7)$ & $2(0)$ & 36 \\
\hline Total Percentage (\%) & & & $\mathbf{6 5}$
\end{tabular}

The absolute number of cases relate to the following order: $\mathrm{x}(\mathrm{y}): \mathrm{x}=$ SHPPC team's assessment, $\mathrm{y}=$ parental assessment. Details see legend to Figure 1.

most frequent combinations included pain and dyspnea (24\%), pain and nausea/vomiting (24\%), agitation and dyspnea (21\%), and agitation and pain (21\%). In $82 \%$ of the cases, three or more symptoms were reported.

\section{Symptom Perception by the SHPPC Team}

The evaluation of symptom perception did not distinguish between the different professions within the SHPPC team. On average, parents reported successful symptom perception by the SHPPC team in $95 \%$ of the cases. Insufficient symptom perception occurred only for two patients who were mainly suffering from nausea/vomiting, seizures, and agitation.

According to the chart review, all symptoms which the parents had been aware of except one (gastrooesophageal reflux) were recognized and documented by SHPPC team members. However, agreement on symptom severity was documented in only $65 \%$ of the cases (see Table 1). According to the parents' perception the members of the SHPPC team mostly underestimated symptom severity. Parental and SHPPC team members' agreement on the successfulness of symptom treatment was documented in $92 \%$ of the cases.

\section{Symptom Treatment}

Successful treatment was mainly reported for the management of "pain" (85\% of reported cases), "seizures" (83\%), "agitation" (75\%), and "nausea/vomiting" $(60 \%)$. Treatment of the frequent and severe symptom "dyspnea" (40\%) and other less frequently mentioned symptoms was less successful. On average, successful symptom treatment was reported in $71 \%$ of cases. In seven cases of unsuccessful symptom treatment, children were admitted to a hospital (18\% of overall cohort). Figure 2 shows the main outcome measures in correlation to the successfulness of symptom treatment. No significant differences were noted.

\section{Perception and Treatment}

The quality of symptom perception by the SHPPC team significantly correlated with the quality of symptom treatment ( $p<0.0001, n=38$ ), both according to parental assessment. There was no correlation between symptom severity and successful symptom treatment ( $p=0.483, n=38$ ). Symptom perception was the only variable predicting the quality of symptom treatment during the child's EOLphase (see Table 2).

\section{Symptom Perception and Quality of Care}

During the period in which the SHPPC team was involved, the parents' distress caused by the children's

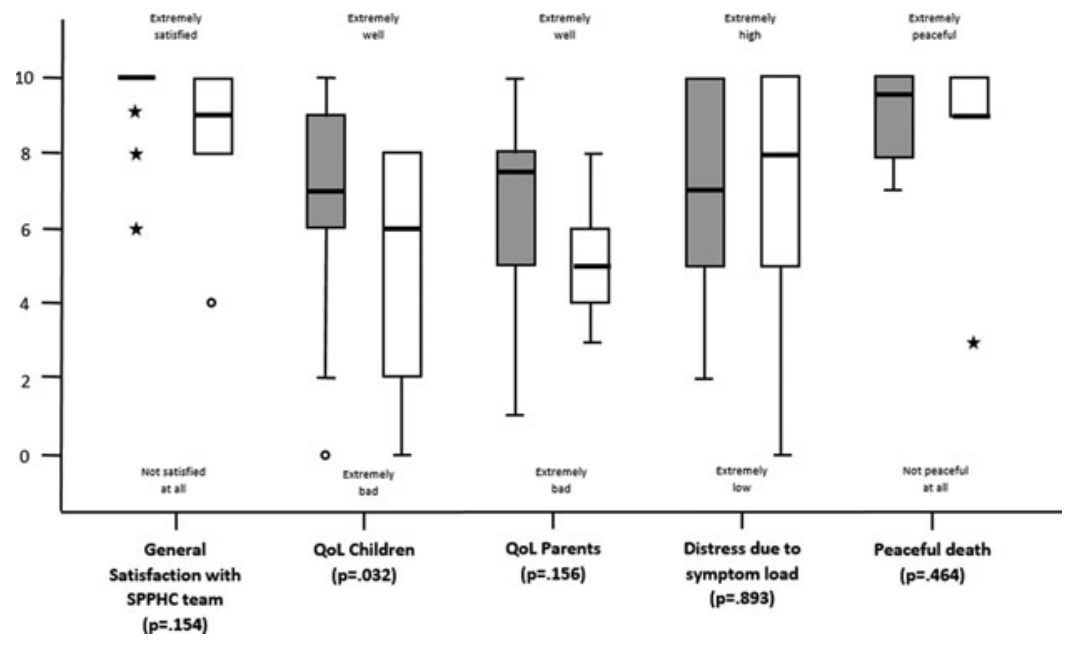

Fig. 2. Box-Plot-Diagrams on different items assessed by parents (NRS $0-10$ ) in a cohort of children $(n=38)$ cared for by the SPPHC team with (grey boxplots; $n=27$ ) and without (white boxplots; $n=11$ ) successful symptom treatment. 
Table 2. Predictive Values for "Quality of Symptom Treatment" according to parental assessment $(n=38)$

\begin{tabular}{lc}
\hline \hline & $\boldsymbol{R}^{\mathbf{2}}$ \\
\hline ACT-Group $^{2}$ & 0.147 \\
Age & 0.105 \\
Communication within local caring team & 0.168 \\
Duration of Contact with PPHC team & 0.077 \\
Information giving through PPHC team & 0.146 \\
Usage of 24/7 medical on-call service & 0.134 \\
Symptom perception & 0.612 \\
Symptom strength & 0.195 \\
\hline
\end{tabular}

${ }^{1}$ Adjusted $R^{2}$-values of performed regression analysis are depicted.

${ }^{2} \mathrm{ACT}=$ Life-threatening and life-limiting conditions can be classified into four ACT groups.

symptom load significantly decreased $(p<0.005)$ and the children's quality-of-life as perceived by the parents significantly improved ( $p<0.005)$. The parents' self-reported quality-of-life at the time of the survey was rated at a median of 5 [NRS $0-10$, mean 5.8, range 1-10]. Overall satisfaction of parents with the care provided by the SHPPC team was extremely high with a median of 10 [NRS $0-10$, mean 9.1, range 4-10]. Even in cases with unsuccessful symptom control, parents rated the child's death as highly peaceful [NRS $0-10$, mean 8.4, median 9, range $0-10]$. In their comments to the questionnaires, parents underlined in $84 \%$ of cases that the SHPPC team significantly contributed to a general decrease in their physical and psychological distress by simply addressing and explaining all existing symptoms and possible barriers to effective symptom management.

A mother of a three-year-old boy said: "Through the involvement of the pediatric palliative home care team, I really felt relieved. Finally, there was somebody to whom I could talk, not only about our child's situation but also about the hopelessness of his case since the diagnosis had been made; what it meant to me, to my husband, and to the whole family; and in which way we could be supported." A father of a four-months-old girl stated: "It was through the periodic visits of the pediatric palliative home care team that my wife and I began to trust that there was somebody who understood our needs. In the beginning, we were afraid that our baby would only be treated for pain, but they explained us how the accompanying symptoms, such as obstipation, occur and what we can do against them. This was really helpful." Moreover, the majority of parents (82\%) stated that the presence and/or the $24 / 7$ service of a specialized SHPPC team significantly contributed to the peacefulness of the dying phase. However, in the two cases of insufficient symptom perception, parents were less satisfied with the care provided [NRS: 6 points]. The concordant assessment of symptom severity between SHPPC team members and parents significantly correlated with the parental quality-oflife $(p=0.041)$ as well as with the parents' general satisfaction with the SHPPC team $(p=0.037)$.

\section{DISCUSSION}

Little data is available on pediatric palliative care and symptom management, particularly in home care settings (Hendricks-Ferguson, 2008; Wolfe et al., 2002). Most published studies depict symptoms of oncological patients in a hospital setting with pain usually being one of the most dominant symptoms (Goldman et al., 2000; Kreicbergs et al., 2005; Pritchard et al., 2008; Wolfe et al., 2000). In our unselected setting involving children with a variety of lifelimiting diseases, dyspnea was at least as important as pain, and more difficult to treat. Depending on the age and the communication abilities of the child, symptoms such as pain and dyspnea can be diagnosed less easily when compared to adults, while other symptoms like nausea often remain underdiagnosed (Kreicbergs et al., 2005). Moreover, the underlying causes of the symptoms might be multi-factorial, and cannot always be clearly assigned to the disease or, for example, to the side effects of medication. Several parents in our study expressed the need to address all existing symptoms, not only pain and/or dyspnea. Thus, it is necessary to broaden the scope and intensify the research concerning the assessment of all potential symptoms in children in palliative care and to develop appropriate training possibilities for HCPs (Bagatell et al., 2002; Michelson et al., 2009; Steele et al., 2008).

The same applies to symptom treatment strategies. In our study, approximately two-thirds of cases reported an overall successful treatment, particularly with respect to pain, seizures, and agitation. However, it can be considerably more difficult to alleviate other symptoms like fatigue, diarrhea or constipation, as various other studies have also demonstrated (Goldman et al., 2006; Poltorak \& Benore, 2006; Ullrich et al., 2007).

Our study indicates that successful symptom treatment may not be the only crucial variable influencing main outcome measures, such as satisfaction with care, quality-of-life of children and parents, or peacefulness of the dying phase. Concordant symptom perception between parents and HCPs appears to be a pivotal factor predicting both satisfaction with the caring team and parental quality-of-life.

Our findings are consistent with the study of Wolfe et al. (2000) who described a staggering difference 
between the parents' and the healthcare providers' perception of symptoms during the dying phase, that might contribute to the child's suffering. In our study, parents highly appreciated adequate advance communication about possible distressing symptoms that might arise during the last days of their child's life.

The fact that the SHPPC team recognized nearly all symptoms reported by the parents, and that the team predominantly evaluated these symptoms in concordance with the parents, could further emphasize the significance of appropriate teamwork among all involved parties. On the other hand, the SHPPC team members' inconsistent classification of symptom severity, especially for symptoms like poor appetite and fatigue, might be due to an attitude that defines certain symptoms as "expected" or "inevitable." This attitude ignores the need of patients and parents that these symptoms are at least acknowledged and explained by HCPs.

As shown by Poltorak and Benore (2006), appropriate symptom perception can be seen as a crucial part of a complex network of various psychological factors, which may contribute to a decrease in fear and anxiety, as well as an improvement of overall coping. Together with our data, these findings underscore the fundamental importance of symptom perception, acknowledgement and explanation for the quality of pediatric palliative care.

The high rate of successful symptom perception in our study could be due to better preconditions for symptom perception at home compared to hospital settings, since parents as the main caregivers at home, are also the direct communication partners of the SHPPC team. As Dussel et al. (2009) showed, careful listening to families' statements on symptoms increases the children's chance to spend their last days at their preferred location.

Our study has a number of limitations. Because of the retrospective design, our data are based on the parents' recollections, which may change over time. Quality-of-life, an important but not the most crucial variable in our analysis, has not exclusively been measured with standardized tools, and may thus not be represented precisely enough. In addition, the parents' responses might be subject to social desirability bias. The absolute numbers are small, which limits generalizability, particularly as this is a single-center study. We strived to minimize sampling errors by contacting all consecutive families who were in the care of the SHPPC team during a specified period.

\section{CONCLUSION}

Adequate symptom management in pediatric palliative care is a major challenge for all healthcare professionals involved. Even though successful treatment is not always possible, successful symptom perception can be achieved in the vast majority of cases, and is in itself a powerful determinant of the perceived quality of care. Our results suggest that careful listening to the parents reporting on their child's symptoms could help prevent suffering and improve parent satisfaction with palliative care as well as their own quality-of-life. This needs to be verified in prospective studies aimed at further improving the effectiveness of symptom perception strategies in pediatric palliative care.

\section{DISCLOSURES}

For all authors, no competing financial interests exist.

\section{ACKNOWLEDGMENTS}

We would like to express our gratitude to the families who shared their experiences in the hope of improving SHPPC services. We are also grateful to Holly G. Prigerson, $\mathrm{PhD}$ (Center for Psychooncology \& Palliative Care Research, Dana-Farber Cancer Institute, Brigham \& Womens' Hospital, Harvard Medical School) and Ulrika Kreicbergs, RN, $\mathrm{PhD}$ (Department of Woman \& Child Health Astrid Lindgren Children's Hospital, Karolinska Institutet, Stockholm, Sweden) for their support in the preparatory stage of the study.

\section{REFERENCES}

Bagatell, R., Meyer, R., Herron, S., Berger, A. and Villar, R. (2002). When children die: a seminar series for pediatric residents. Pediatrics, 110, 348-53.

Bona, K., Bates, J. and Wolfe, J. (2011). Massachusetts' Pediatric Palliative Care Network: successful implementation of a novel state-funded pediatric palliative care program. Journal of Palliative Medicine, 14, $1217-23$.

Carter, B. S., Howenstein, M., Gilmer, M. J., Throop, P., France, D. \& Whitlock, J. A. (2004). Circumstances surrounding the deaths of hospitalized children: opportunities for pediatric palliative care. Pediatrics, 114, e361-6.

Dangel, T. (2002). The status of pediatric palliative care in Europe. Journal of Pain and Symptom Management, $24,160-165$.

Drake, R., Frost, J. \& Collins, J. J. (2003). The symptoms of dying children. Journal of Pain and Symptom Management, 26, 594-603.

Dussel, V., Kreicbergs, U., Hilden, J. M., Watterson, J., Moore, C., Turner, B. G., Weeks, J. C. \& Wolfe, J. (2009). Looking beyond where children die: determinants and effects of planning a child's location of death. Journal of Pain and Symptom Management, 37, 33-43.

Ellershaw, J. \& Ward, C. (2003). Care of the dying patient: the last hours or days of life. British Medical Journal, $326,30-4$.

Friedrichsdorf, S. J., Remke, S., Symalla, B., Gibbon, C. \& Chrastek, J. (2007). Developing a pain and palliative 
care programme at a US children's hospital. International Journal of Palliative Nursing, 13, 534-42.

Galloway, K. S. \& Yaster, M. (2000). Pain and symptom control in terminally ill children. Pediatric Clinics of North America, 47, 711-46.

Goldman, A. \& Chir, B. (2000). Symptoms and suffering at the end of life in children with cancer [Letter to the editor]. New England Journal of Medicine, 342, 1998.

Goldman, A., Hewitt, M., Collins, G. S., Childs, M. \& Hain, R. (2006). Symptoms in children/young people with progressive malignant disease: United Kingdom Children's Cancer Study Group/Paediatric Oncology Nurses Forum survey. Pediatrics, 117, e1179-86.

Hendricks-Ferguson, V. (2008). Physical symptoms of children receiving pediatric hospice care at home during the last week of life. Oncology Nursing Forum, 35, e108-15.

Hilden, J. M., Emanuel, E. J., Fairclough, D. L., Link, M. P., Foley, K. M., Clarridge, B. C., Schnipper, L. E. \& Mayer, R. J. (2001). Attitudes and practices among pediatric oncologists regarding end-of-life care: Results of the 1998 American Society of Clinical Oncology survey. Journal of Clinical Oncology, 19, 205-12.

Himelstein, B. P., Hilden, J. M., Boldt, A. M. \& Weissman, D. (2004). Pediatric palliative care. New England Journal of Medicine, 350, 1752-62.

Kreicbergs, U., Valdimarsdottir, U., Onelov, E., Bjork, O., Steineck, G. \& Henter, J. I. (2005). Care-related distress: a nationwide study of parents who lost their child to cancer. Journal of Clinical Oncology, 23, 9162-71.

Liben, S., Papadatou, D. \& Wolfe, J. (2008). Paediatric palliative care: challenges and emerging ideas. The Lancet, $371,852-864$.

Michelson, K. N., Ryan, A. D., Jovanovic, B. \& Frader, J. (2009). Pediatric residents' and fellows' perspectives on palliative care education. Journal of Palliative Medicine, 12, 451-7.

Moody, K., Siegel, L., Scharbach, K., Cunningham, L. \& Cantor, R. M. (2011). Pediatric palliative care. Primary Care, 38, 327-61, ix.

Morgan, E. R. \& Murphy, S. B. (2000). Care of children who are dying of cancer. New England Journal of Medicine, $342,347-8$.

Pierucci, R. L., Kirby, R. S. \& Leuthner, S. R. (2001). Endof-Life Care for Neonates and Infants: The Experience and Effects of a Palliative Care Consultation Service. Pediatrics, 108, 653-660.

Poltorak, D. Y. \& Benore, E. (2006). Cognitive-behavioral interventions for physical symptom management in pediatric palliative medicine. Child \& Adolescent Psychiatric Clinics of North America, 15, 683-91.

Pritchard, M., Burghen, E., Srivastava, D. K., Okuma, J., Anderson, L., Powell, B., Furman, W. L. \& Hinds, P. S. (2008). Cancer-related symptoms most concerning to parents during the last week and last day of their child's life. Pediatrics, 121, e1301-9.

Steele, R., Bosma, H., Johnston, M. F., Cadell, S., Davies, B., Siden, H. \& Straatman, L. (2008). Research priorities in pediatric palliative care: a Delphi study. Journal of Palliative Care, 24, 229-39.

Swinney, R., Yin, L., Lee, A., Rubin, D. and Anderson, C. (2007). The role of support staff in pediatric palliative care: Their perceptions, training, and available resources. Journal of Palliative Care, 23, 44-50.

Toce, S. \& Collins, M. A. (2003). The FOOTPRINTS model of pediatric palliative care. Journal of Palliative Medicine, 6, 989-1000.

Ullrich, C. K. \& Mayer, O. H. (2007). Assessment and management of fatigue and dyspnea in pediatric palliative care. Pediatric Clinics of North America, 54, 735-56, xi.

Vollenbroich, R., Duroux, A., Grasser, M., Brandstatter, M., Borasio, G. D. \& Fuhrer, M. (2012). Effectiveness of a pediatric palliative home care team as experienced by parents and health care professionals. Journal of Palliative Medicine, 15, 294-300.

WHO. (1998). WHO definition of palliative care.

Wolfe, J., Friebert, S. \& Hilden, J. (2002). Caring for children with advanced cancer integrating pallaitive care. Pediatric Clinics of North America, 49, 1043-1062.

Wolfe, J., Grier, H. E., Klar, N., Levin, S. B., Ellenbogen, J. M., Salem-Schatz, S., Emanuel, E. J. \& Weeks, J. C. (2000). Symptoms and suffering at the end of life in children with cancer. New England Journal of Medicine, $342,326-33$

Zhukovsky, D. S., Herzog, C. E., Kaur, G., Palmer, J. L. \& Bruera, E. (2009). The impact of palliative care consultation on symptom assessment, communication needs, and palliative interventions in pediatric patients with cancer. Journal of Palliative Medicine, 12, 343-9. 